\title{
Neurosurgical robotics: a review of brain and spine applications
}

\author{
Chris S. Karas · E. Antonio Chiocca
}

Accepted: 28 November 2006/ Published online: 27 February 2007

(C) Springer London 2007

\begin{abstract}
Neurosurgery has traditionally been at the forefront of advancing technologies, adapting new techniques and devices successfully in an effort to increase the safety and efficacy of brain and spine surgery. Among these adaptations are surgical robotics. This paper reviews some of the more promising systems in neurosurgical robotics, including brain and spine applications in use and in development. The purpose of the discussion is twofold-to discuss the most promising models for neurosurgical applications, and to discuss some of the pitfalls of robotic neurosurgery given the unique anatomy of the brain and spine.
\end{abstract}

Keywords Minimally invasive $\cdot$ Neurosurgery ·

Robot · Robotic technology

\section{Introduction}

Neurosurgery has traditionally been at the forefront of advancing technologies, adapting new techniques and devices successfully in an effort to increase the safety and efficacy of brain and spine surgery. Among these adaptations are surgical robotics. That surgical robots have not found widespread clinical utilization in neurosurgical procedures is debatable, because the term "robot" itself has several definitions [1]:

C. S. Karas $(\bowtie) \cdot$ E. A. Chiocca

Department of Neurosurgery,

The Ohio State University Medical Center,

410 W. 10th Avenue, Columbus,

OH 43201, USA

e-mail: ckaras@yahoo.com
1. A mechanical device that sometimes resembles a human and is capable of performing a variety of often complex human tasks on command or by being programmed.

2. A machine or device that operates automatically or by remote control.

3. A person who works mechanically without original thought, especially one who responds automatically to the commands of others.

In recent years, it is clear that technologies advanced by the field of robotics have been incorporated into the operating room through microscopy, navigation, instrumentation, optics, and imaging [2]. The use of a mechanical device, whether through automation or remote control, to ultimately manipulate the instruments directly in contact with a patient is relatively new to brain and spine surgery, however. Since Kwoh et al. attempted a robotic brain biopsy in the late 1980s, growing interest in this field and its potential clinical benefits has encouraged the development of multiple systems [3]. As with all novel instrumentation, the role of these systems must be clearly defined. Among neurosurgeons this is particularly challenging, because the concepts of manual microsurgical techniques are already embedded effectively and successfully in standard practice. Approaching central nervous system pathology within millimeters through small working channels surrounded by vital tissue almost defines the subspecialty. Integration of surgical robotics is, therefore, an interesting dilemma. Although its theoretical advantages seem most suited to neurosurgical disease, the state of the art has not yet matched the theory. Despite these practical hindrances, advances coupling clinical, and scientific discovery, 
continue to expand the notion of what is possible. This paper reviews some of the more promising systems in neurosurgical robotics, including brain and spine applications, in use and in development (Table 1).

\section{Brain}

Several robotic solutions have been developed to address the specific challenges associated with interventions on the brain [2-6]. Deep pathology requiring manipulation of or direct trauma to the parenchyma has inspired devices which may minimize damage to normal tissue $[3,6]$. Although this is not meant to serve as a review of surgical robotics in general, an understanding of the subtypes of system available is helpful. Nathoo et al. eloquently propose a classification based on the robot-surgeon interaction [2]. Three systems are described. The first is a supervisory-controlled robotic system in which the robotic intervention is preplanned and programmed and then supervised by the surgeon as it carries out its programmed movements autonomously. The second is a robotic telesurgical system in which the robot is manipulated by the surgeon in real time through remote control, with limited feedback to the operator. The third is a shared control system in which the surgeon directly controls the movements of the robot as the robot enhances the surgeon's skills through dexterity enhancement, a term which generally describes mechanical solutions to human limitations, including physiologic tremor reduction.

As already stated, attention has been focused on gaining access to deep pathology or structures (such as the third ventricle) with limited trauma to the normal brain. Coupling these devices, therefore, with imagebased navigation systems and developing controlled, precise target-acquisition capabilities have been crucial advances in attempting intracranial procedures. In general, with these resources, existing models focus their technology on specific tasks.

Among the simplest and most widely used supervisory-controlled robot is a particular upgrade to the Leksell Gamma Knife radiosurgical system. The Automated Positioning System (APS) (Elekta, Stockholm, Sweden) will adjust the patient's head within a collimator automatically, based on a predetermined stereotactic plan. Several studies have confirmed the benefits of such automation, confirming shorter treatment times, reduced exposure of patients and personnel to radiation, and greater ability to deliver radiation to an increased number of smaller isocenters, thereby reducing the maximum dose to the target $[7,8]$.

The NeuroMate (Integrated Surgical Systems, Sacramento, CA, USA) robotic surgical system was the first FDA-approved robotic device for neurosurgery [4]. Like Minerva (University of Lausanne, Lausanne, Switzerland) which followed, this system involves a passive robotic arm which moves in a preprogrammed direction to a specific site defined by integrated neuronavigation systems for stereotactic biopsy or functional neurosurgical applications [9]. The Minerva project attempted to account for brain shift by placing the robotic arm within a CT scanner to provide real-time image guidance. Safety issues forced the discontinuation of this device [2]. Indications for the NeuroMate continue to expand as imageguidance technology advances. Recent studies have proven its localization and targeting capabilities are comparable with those of standard localizing systems

Table 1 Robotic surgical devices with FDA-approved and experimental neurosurgical applications

\begin{tabular}{|c|c|c|c|c|}
\hline Name & Type & Function & Advantages & Disadvantage \\
\hline $\begin{array}{l}\text { Automated } \\
\text { positioning system }\end{array}$ & $\begin{array}{l}\text { Supervisory } \\
\text { controlled }\end{array}$ & Radiosurgery & Precision & Limited function \\
\hline NeuroMate & $\begin{array}{l}\text { Supervisory } \\
\text { controlled }\end{array}$ & Biopsy, MDS & Precision & Limited function, cost \\
\hline Minerva & $\begin{array}{l}\text { Supervisory } \\
\text { controlled }\end{array}$ & Biopsy & Precision & Limited function, safety issues \\
\hline Evolution 1 & Shared control & $\begin{array}{l}\text { Pedicle screws, ETV, } \\
\text { transsphenoidal }\end{array}$ & $\begin{array}{l}\text { Dexterity } \\
\text { enhancement }\end{array}$ & Lack of sensory feedback, cost \\
\hline da Vinci & Telesurgical & $\begin{array}{l}\text { Urologic, gynecologic, and } \\
\text { general surgery }\end{array}$ & $\begin{array}{l}\text { Dexterity } \\
\text { enhancement }\end{array}$ & $\begin{array}{l}\text { Lack of sensory feedback, not equipped } \\
\text { for bone/disc work }\end{array}$ \\
\hline NeuRobot & Telesurgical & Tumor resection & $\begin{array}{l}\text { Dexterity } \\
\text { enhancement }\end{array}$ & Lack of sensory feedback, cost \\
\hline $\begin{array}{l}\text { Cyberknife, } \\
\text { RoboCouch }\end{array}$ & $\begin{array}{l}\text { Supervisory } \\
\text { controlled }\end{array}$ & Radiosurgery & Precision & Limited function \\
\hline SpineAssist & $\begin{array}{l}\text { Supervisory } \\
\text { controlled }\end{array}$ & Pedicle screws & Precision & Limited function, cost \\
\hline
\end{tabular}


[10]. Varma et al. achieved good accuracy with a frameless application of this system in microelectode placement for treatment of Parkinson's disease [11].

The Evolution 1 robotic system (Universal Robot Systems, Schwerin, Germany) has been tested for several neurosurgical applications. Pedicle screw placements and endoscope-assisted transphenoidal pituitary ademona resections, although successful, were deemed by those who attempted these applications to be too cumbersome and time-consuming to justify their use [12]. More recently this system has been used for endoscopic third ventriculostomy (ETV) in six patients with hydrocephalus secondary to aqueductal stenosis [13]. Specifically, the robotic arm was used to precisely and reliably guide an endoscope to visualize the floor of the third ventricle. The ventriculostomy was performed manually by the surgeon through working channels in the endoscope, which was held rigidly by the robot. Theoretical advantages of this system over surgeon-alone ETV are precision targeting through image-guidance coupling and dexterity enhancement, which eliminates micro movements of a hand-held scope. Thus far there is no evidence supporting a clinical or outcome benefit of robotic over manual ETV, despite the measured differences.

Beyond interventions requiring a single instrument or endoscope-stabilization solutions, telesurgical systems with multiple arms for both variable instrumentation and endoscopy are currently available in other specialties [2, 14]. The Neurobot telerobotic surgical system has been used successfully in complex procedures requiring simultaneous retraction and dissection [15]. Goto et al. describe a robot-assisted craniotomy in which the NeuRobot is used to resect superficial portions of an intraaxial tumor on a live human subject, citing dexterity enhancement as one of the potential advantages [16]. At our institution several da Vinci surgical systems are available for both clinical use and research purposes. It has become standard instrumentation for prostatectomy and other urological procedures, and is FDA-approved for general and gynecologic surgery also. Given its tremor reduction, motion scaling capabilities, multiple working arms, and patented Endowrist (Intuitive Surgical, Sunnyvale, CA, USA) technology which enables for full range of motion at the instrument head comparable with that of the human wrist, this device was tested at our institution for several neurosurgical procedures also. In our experience with cadaveric trials of end-to-end ulnar nerve reanastomosis, lumbar discectomy, intradural spinal dissection, and complex intraventricular surgery, significant obstacles to brain and spine applications still remain (Oral Presentation, AANS/CNS Section on Pediatrics, Denver, USA, 2006).

These obstacles, however, do provide insight into some of the necessities of robotic neurosurgery, which require both software and hardware changes. Specifically, the traditional endoscope with working channels allows for one tract through normal tissue to the ventricles rather than multiple tracts to accommodate instrumentation. This traditional model coupled with Endowrist technology may provide the added benefit of a greater range of motion within the ventricular system, which is otherwise impossible to achieve manually. Robotic devices focused on accurate localization may also move, or be manipulated, in such a way as to precisely acquire a target at a deep location at the expense of normal tissue at a more superficial level. For example, an endoscope positioned robotically to view the floor of the third ventricle may pivot dangerously at the cortex or foramen of Monroe and fornix. Docking after target acquisition, therefore, with continued mobility only distally is ideal. Finally, a clear disadvantage within all categories of surgical-robotic models is the lack of feedback to the operator. Although visual feedback has improved significantly with advances in optics and image-guidance, other sensory feedback is lagging. Position, velocity, or acceleration of the instruments may be recognized through a combination of visual cues and, for telesurgical or shared-surgical models, proprioceptive cues. Without complete sensory feedback, however, other significant sensations are lost, including force on adjacent structures or characteristics of manipulated tissues, for example compliance, texture, pulsatility, or elastisticy. Active research in this aspect of robotics continues and will be crucial in the integration of these systems into neurosurgery given the arguably absolute necessity of such feedback when operating within the central nervous system $[17,18]$.

\section{Spine}

Several robotic systems have been developed to address the challenges encountered in spinal surgery. As with brain applications, these devices are enhanced significantly by advances in intraoperative imageguidance. In general, research in this area has focused on accurate placement of spinal instrumentation, citing the theoretically increased accuracy that robotics offers $[16,19,20]$. In radiosurgery, robotic solutions to spine motion with respiration have also been extremely useful [21]. 
As with intracranial radiosurgical applications, the most common robotic subtype in spinal stereotactic radiosurgery is a supervisory-controlled system. Cyberknife (Accuracy, Sunnyvale, CA, USA) relies on a predetermined plan which targets spinal pathology for focused beam radiotherapy. By use of feedback mechanisms this system can adjust its trajectory to correct for patient movement, most of which result from respiration. This novel use of robotics has been expanded to intracranial use also, given the possibility of brain shift. A recent addition to the Cyberknife system is the RoboCouch Patient Positioning System (Accuracy), which uses similar technology to reposition the patient during the course of treatment.

Other supervisory-controlled systems have been developed for conventional spinal surgery also [22, 23]. Specifically, devices coupled with image-guided navigation systems have been tested for accurate pedicle screw placement. Most recently, Lieberman et al. tested the SpineAssist (MAZOR Surgical Technologies, Caesarea, Israel) miniature robot for both pedicle and translaminar facet screw placement [23]. Again, this device consists of a passive arm which mounted on a fixed part of the axial skeleton. Motion of the robotic arm is defined by preoperatively planned screw trajectories, and is supervised by the surgeon. This and other robots with similar functionality have been tested successfully on human subjects, and the SpineAssist device is currently FDA-approved for spinal instrumentation.

As stated previously, we have tested several procedures with the da Vinci Surgical System at our institution, including lumbar discectomy, and intradural dissection. Because of the focused function of most robotic devices, it is clear that operations requiring both bony and soft tissue manipulation at different stages would also require human intervention at some point or multiple limited-function robots. Even the multifaceted design of the da Vinci telesurgical robot with multiple arms is limited in spinal surgery. The range of forces provided by this device, while adequate for abdominal or gynecologic surgery, does not enable use of a drill for bone remodeling, nor does it facilitate extraction of disc material. Without this capability, discectomy is nearly impossible, and intradural intervention requires conventional manual laminectomy. In a cadaveric study, after laminectomy, the da Vinci robot was used to open and close the dura and to separate nerve roots in the cauda equina from the filum (unpublished work, 2006). These maneuvers were performed with relatively little trauma despite only visual feedback.

\section{Conclusion}

Surgical robots have clearly affected the practice of neurosurgery through several FDA-approved devices, most notably in the realm of radiosurgery. It is clear, however, that while the field of surgical robotics advances, attention must be given to the details of brain and spine surgery and surgical anatomy. Integrations of new focused technologies then can be adapted more easily into the neurosurgeon's already highly specialized operating environment. Creating the future of dexterity enhancement, automation, and sensory feedback, is of most value to surgical robotics if it can be studied in the context of each specialty. The robots most widely used in neurosurgery have been products of this contextual research, which concentrated on central nervous system-specific solutions. Attempts to adapt other instrumentation for neurosurgical use have proven to be less effective.

\section{References}

1. The American Heritage ${ }^{\circledR}$ (2000) Dictionary of the English Language, 4th edn. Houghton Mifflin Company, Boston, MA, USA

2. Nathoo N, Cavusoglu MC, Vogelbaum MA et al (2005) In touch with robotics: neurosurgery for the future. Neurosurgery 56:421-433

3. Kwoh YS, Hou J, Jonckheere EA, Hayati S (1988) A robot with improved absolute positioning accuracy for CT guided stereotactic brain surgery. IEEE Trans Biomed Eng 35(2):153-160

4. Benabid AL, Cinquin P, Lavelle $S$ et al (1987) Computer driven robot for stereotactic surgery connected to CT scan, and magnetic resonance imaging: technological design and preliminary results. Appl Neurophysiol 50:153-154

5. Benabid AL, Hoffman D, Munari C et al (1992) Potential use of robots in endoscopic neurosurgery. Acta Neurochir Suppl 54:93-97

6. Drake JM, Joy M, Goldberg A et al (1991) Computer and robot-assisted resection of thalamic astrocytomas in children. Neurosurgery 29:27-31

7. Regis J, Hayashi M, Porcheron D et al (2002) Impact of the model $\mathrm{C}$ and automatic positioning system on gamma knife radiosurgery: an evaluation in vestibular schwannomas. J Neurosurg 97(Suppl 5):588-591

8. Tlachacova D, Schmitt M, Novotny J et al (2005) A comparison of the gamma knife model $\mathrm{C}$ and the automatic positioning system with leksell model B. J Neurosurg 102(Suppl):25-28

9. Glauser D, Frankhauser H, Epitaux M et al (1995) Neurosurgical robot Minerva: first results and current developments. J Image Guid Surg 1:266-272

10. Li QH, Zamorano L, Pandya A et al (2002) The application accuracy of the neuromate robot-a quantitative comparison with frameless and frame-based surgical localization systems. Comput Aided Surg 7(2):90-98

11. Varma TR, Eldridge PR, Forster A et al (2003) Use of the neuromate robot in a frameless mode for movement disorder surgery. Stereotact Funct Neurosurg 80(1-4):132-135 
12. Nimsky J, Rachinger H, Iro R, Fahlbusch (2004) Adaptation of a hexapod-based robotic system for extended endoscopeassisted transsphenoidal skull base surgery. Minim Invasive Neurosurg 47:41-46

13. Zimmerman M, Krishnan R, Raabe A (2004) Robot-assisted navigated endoscopic ventriculostomy: implementation of a new technology and first clinical results. Acta Neurochir 146:697-704

14. Stoianovici D (2000) Robotic surgery. World J Urol 18:289295

15. Hongo K, Kobayashi S, Kakizawa Y et al (2002) Neurobot: telecontrolled micromanipulator system for minimally invasive microneurosurgery-preliminary results. Neurosurgery 51:985-988

16. Goto T, Hongo K, Kakizawa Y et al (2003) Clinical application of robotic telemanipulator system in neurosurgery. J Neurosurg 99:1082-1084

17. Gray BL, Fearing RS (1996) A surface micromachined microtactile sensor array. Presented at the IEEE international conference on robotics and automation, Minneapolis
18. Moy G, Wagner C, Fearing RS (2000) A compliant tactile display for teletaction. Presented at the IEEE international conference on robotics and automation, San Francisco

19. Garcia-Ruiz A, Gagner M, Miller JH (1998) Manual versus robotically assisted laparoscopic surgery in the performance of basic manipulation and suturing tasks. Arch Surg 133:957961

20. Taylor RH, Jensen P, Whitcomb LL et al (1999) A steadyhand robotic system for microsurgical augmentation. Int $\mathbf{J}$ Robot Res 18:1201-1210

21. Adler JR, Murphy MJ, Chang SD et al (1999) Image-guided robotic radiosurgery. Neurosurgery 44:1299-1307

22. Lieberman IH, Togawa D, Kayanja MM et al (2006) Bone-mounted miniature robotic guidance for pedicle screw and translaminar facet screw placement: part 1-technical development and a test case result. Neurosurgery 59:641-648

23. Chop WW, Green B, Levi A (2000) Fluoroscopic guided targeting system with a robotic arm for pedicle screw insertion. Neurosurgery 47:872-878 